\title{
Finite element modeling of large radius bending operation
}

\author{
Keywords Bending, Sheet metal, Springback, Finite Element
}

Modeling, High-strength steel, Aerens law

\begin{abstract}
In this work, this method is applied to the prediction of large radius air bending of high-strength steels. Three distinct formulations are used for the prediction: plane, shell, and solid. Appropriate mesh parameters and material implementation are used in order to obtain high prediction accuracy and to minimize the calculation time. A new law for the approximation of the hardening behavior of high-strength steels is introduced and further used for the minimization of the prediction error. Contact points position, springback, bending force and bend allowance are used for the comparison between experimental and simulation data. Obtained results show that the plane model is not a suitable option for the modeling of large radius air bending, due to an instable behavior with respect to the number of elements through the thickness. Shell and solid formulations, however, provide high accuracy prediction for the considered bending characteristics with comparable predictive quality.
\end{abstract}

\section{Introduction}

Industrial applications of air bending conventionally involve small radius tooling (Figure 1a). Large radius air bending recently has attracted more attention because of the increasing usage of high-strength steels. High-strength steels have superior load bearing characteristics, however this quality usually results in very limited ductility. In order to be able to form high-strength steels, a reduced degree of deformation should be imposed during the forming stage, for instance by using larger punch radii (Figure 1b). Industry looks towards bending with large radii punches as the solution for the limited ductility of high-strength steels. However, large radius bending brings about several difficulties, such as the multi-breakage effect. The peculiarities of the large radius bending are described in detail in [1].

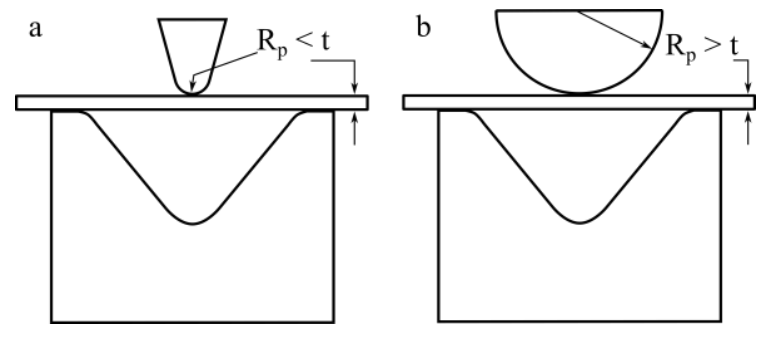

Figure 1 - Two types of bending: conventional (a) and large-radius (b)

In order to reduce the cost and time of production, an accurate prediction of air bending should be used. The prediction of air bending has been attempted by means of various approaches, including analytical prediction [2-4], regression modeling [5,6], and neural networks [5,7]. Another popular option is the prediction of bending characteristics by means of the finite element method. Vorkov et al. used finite element analysis for the determination of various bending characteristics [8,9]. Ledentsov et al. [10] applied the model-adaptive technique for meshing of a part during forming. Arola et al. [11] used the LS-Dyna solver to predict the bendability of the high-strength steels. Eggertsten and Mattiasson [12] studied different hardening mechanisms that affect the accuracy of springback predictions.

This contribution focuses on the finite element prediction of large radius bending. Despite prior efforts in springback calculation [13], scientific literature on the behavior of thick plates (more than four millimeters) of high-strength steels with large radii punches is limited. 
Since large radius air bending is applied virtually only to high-strength steels, two available materials of this type have been selected for the current study: Strenx 1300 (plate thicknesses 4 and $6 \mathrm{~mm}$ ) [14], and Strenx $700 \mathrm{MC}$ (plate thicknesses 4 and $6 \mathrm{~mm}$ ) [15]. Different parameters can be used for the assessment of the prediction model accuracy. In this contribution, the following parameters are used: the contact points position (Figure 2a), the bending force, the springback (Figure 2b), and bend allowance or BA (Figure 2c). For a more detailed description of practical measurements of these bending characteristics the reader can refer to [1].
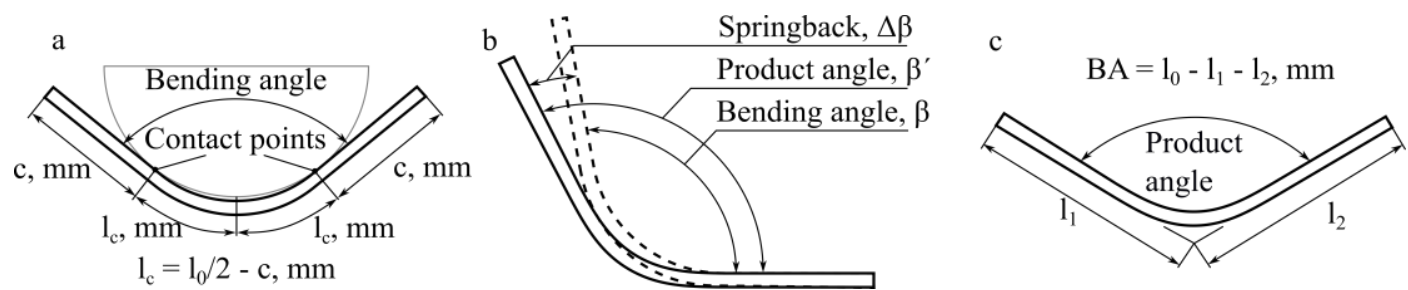

Figure 2 - Characteristics of large radius bending: contact points position (a), springback (b), and bend allowance or BA (c)

The goal of this contribution is to obtain a reliable methodology for constructing an accurate finite element calculation model of large radius bending, which is applicable for a wide range of thicknesses, materials and tooling dimensions.

This contribution is organized as follows. Section 2 provides a general overview of the finite element models. The selection of mesh parameters is addressed in Section 3, and the choice and implementation of the material model are described in Section 4. Section 5 discusses the initial obtained results and compares them to experimental data. A new hardening law for high-strength steels is presented in Section 6, and the approach for selection of the adapted material parameters is described in Section 7. Section 8 discusses the results with adapted material parameters and the final section offers final remarks and conclusions.

\section{Model description}

The finite element simulations are executed using the commercial code Abaqus. The problem is solved in two steps. The first step is the forming stage, and the second step is the springback, where no external loads are applied to a bent plate.

Since the considered problem is naturally not linear with regards to material and geometry, and an implementation of contact interaction is necessary, convergence problems are expected to occur. Therefore, to prevent these issues, the following solution steps have been selected. The forming phase is implemented as an explicit model, which has four sub-steps for the considered bending angles $\left(90^{\circ}\right.$, $110^{\circ}, 130^{\circ}$, and $\left.150^{\circ}\right)$. This scheme of divisions is used to extract springback values for every forming angle from only one explicit model, this approach reduces the calculation time significantly.

An implicit model is used for the springback phase. In this model, only symmetrical boundary conditions are retained, since the calculation of the springback does not require any loading or contact interaction. Springback is calculated for every considered bending angle, where the starting information is extracted from the corresponding frame of the forming step.

Three types of explicit elements and their implicit counterparts are selected for the finite element simulations: 
- a plane element (CPE4R) with second-order accuracy and enhanced hourglass control in plane strain mode;

- a shell element (S4) with second-order accuracy and finite membrane strains;

- a solid element (C3D8) with second-order accuracy and distortion control (length ratio 0.1).

The used in the experimental investigation plates are $60 \mathrm{~mm}$ by $110 \mathrm{~mm}$ for all thicknesses and materials. The bending model that is developed in the current contribution has two planes of symmetry (Figure 3). Therefore, a quarter of the full model (or half in the case of the plane model) is implemented to save computation resources. On the symmetry edges, boundary conditions of corresponding symmetry constraints are imposed. Loading is applied by displacement control. All displacements are taken according to the experimental investigation presented in [1].

The forming tooling (a punch and a die) is modeled as rigid analytical surfaces, because both tools are much more rigid than a formed plate. Interaction between a bent plate and the forming tooling is implemented by means of surface to surface (or line to line in the case of the plane model) sliding contact interaction with the following friction coefficients: 0.3 for Strenx 1300 and 0.2 for Strenx 700 MC. The friction coefficients are set according to the experiments performed by Aerens [2] on coarse and fine materials. Figure 3 shows the conceptual representation of the described model. Figure 4 depicts all three implemented models with different element formulations.

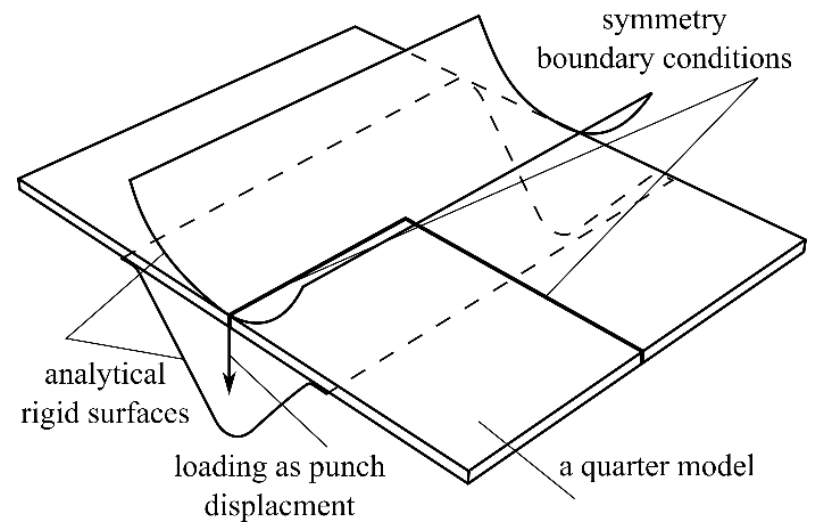

Figure 3 -Conceptual representation of a finite element model with two surfaces of symmetry

The goals of the current work require a large number of simulations to be executed. Thus, an automated procedure is an advisable option to execute the simulations. Therefore, the model creation, the analysis submission and the result treatment have been commanded through a Python macro. 


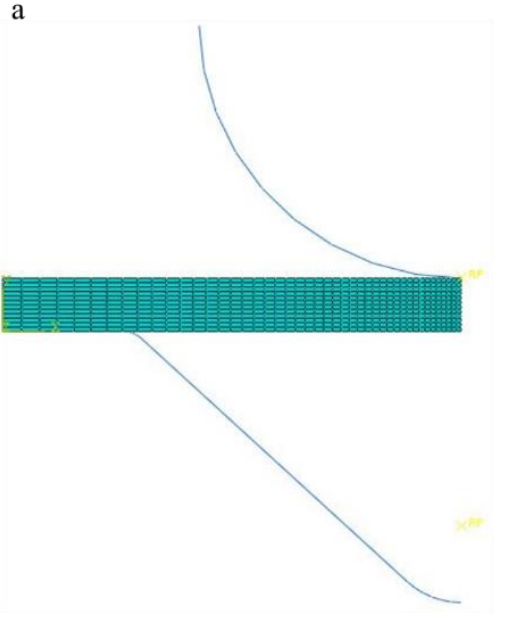

b

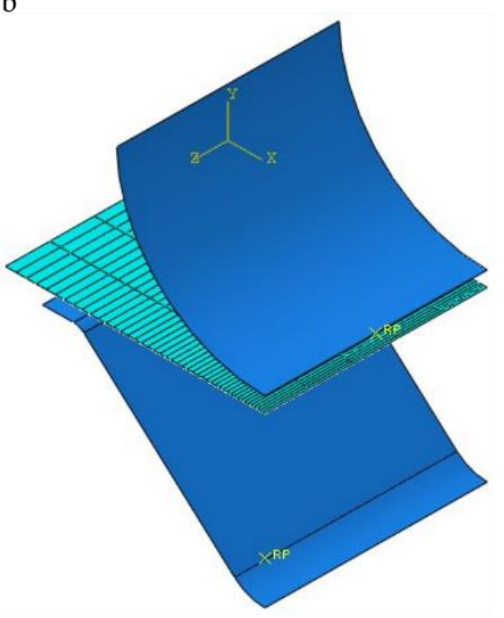

c

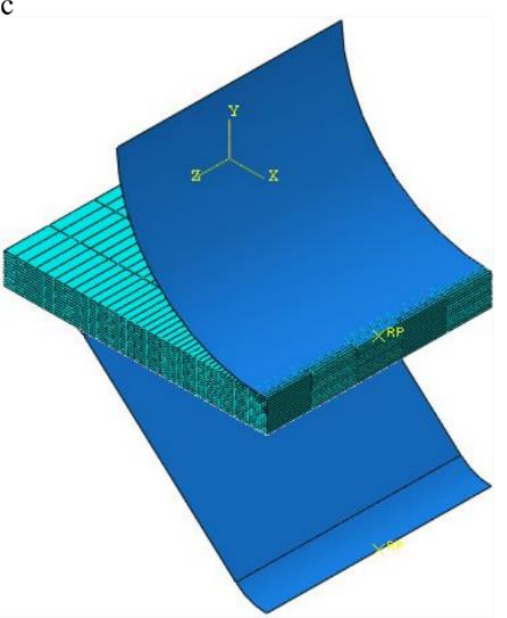

Figure 4-Implemented finite element models: a) plane formulation; $\boldsymbol{b})$ shell formulation; $\boldsymbol{c})$ solid formulation. Thickness: $4 \mathrm{~mm}$; die opening: $50 \mathrm{~mm}$; punch radius: $20 \mathrm{~mm}$

The contact point positions are usually measured along the lines where the maximal contact forces between the punch and the bent plate are applied. Forming angles, springback, and bend allowance are evaluated in a post-processing step (Figure 2). The value of the bending force is taken according to the total value of the frictional and normal reaction forces between a die and a plate.

\section{Mesh parameters}

To set optimal mesh size parameters, a number of test simulation have been performed first, on a case of Strenx $13006 \mathrm{~mm}$ with die opening of $80 \mathrm{~mm}$ and punch radius of $40 \mathrm{~mm}$. This combination is expected to be the worst-case scenario, since the loading scheme changes mostly in this case and there are fewer points to integrate stress correctly for thicker plates.

Springback is the most influential parameter of all bending characteristics: it is very important itself in industrial practice, as it defines the final shape with regard to the product angle (Figure $2 \mathrm{~b}$ ), but it also has a significant influence on the bend allowance [6]. Hence, the springback was selected as the decisive parameter in the mesh selection procedure.

A number of calculations were executed with gradual mesh refinement. The relative difference $\Delta$ between two consecutive calculations with regards to springback $\Delta \beta$ was calculated as follows:

$$
\Delta=\left|\frac{\Delta \beta_{i}-\Delta \beta_{i-1}}{\Delta \beta_{i}}\right|
$$

Since geometrical configuration of the calculation model varies a lot, a threshold was set for elements along length and width of $2.5 \%$ and through the thickness of $1.0 \%$. The smaller value of the threshold for the elements through the thickness is due to its crucial role in the calculation, as explained in the next paragraph. Initial mesh parameters were set as follows: elements along the width -40 elements (with the bias coefficient of four in the direction from the center of a plate), elements along the length -10 elements.

All simulations were performed for the four bending angles: $90^{\circ}, 110^{\circ}, 130^{\circ}$ and $150^{\circ}$. Further, in this contribution, these angles are referred as Angle $90^{\circ}$, Angle $110^{\circ}$, Angle $130^{\circ}$, and Angle $150^{\circ}$. 
The number of elements or integration points through the thickness of the plate was considered the main parameter for the forming calculations. An insufficient number of elements through the thickness sometimes leads to the impression that the correct mesh parameters have been set [16]: this is caused by the oscillatory effect of stress integration through the thickness of a sheet. In order to estimate a sufficient number of elements/integration points through the thickness, simulations from 2 to 20 elements have been performed for all considered element formulations: plane, shell, and solid.

Figure 5 and Figure 6 show the results for the shell and solid models with respect to the elements or integration points through the thickness. For the shell model, 13 integration points are sufficient for $1 \%$ of $\Delta$, and six elements suffice for the solid model. However, in order to compare the models performance further, the higher number of elements/integration points are selected. Further mesh selection procedure is performed with 12 elements for the solid model or 13 integration points for the shell model through the thickness.

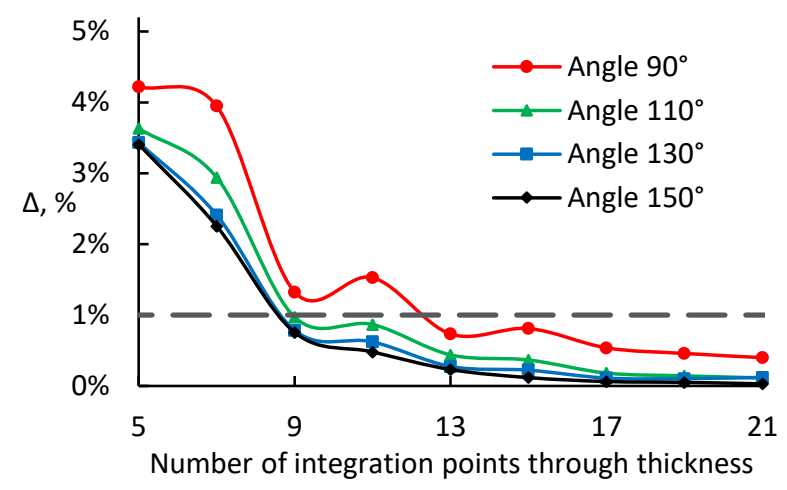

Figure 5 - Percentage springback difference as function of the number of integration points through thickness for the shell model. 13 integration points are sufficient to have less than $1 \%$ of difference

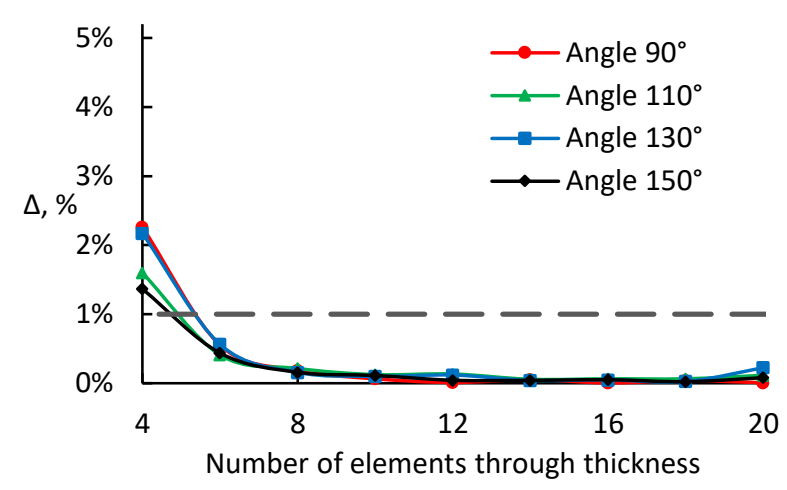

Figure 6-Percentage springback difference as function of the number of elements through thickness for the solid model. Even six elements are sufficient in this case to assure less than $1 \%$ of difference

Through thickness stresses for models with different numbers of elements through the thickness are presented in Figure 7. As it can be seen two elements provide only a rough representation of the stress distribution in the plate (Figure 7a). The model with six elements through thickness already provides a reasonable representation of the stress situation (Figure $7 \mathrm{~b}$ ), and further increase of elements through the thickness will provide even finer stress distributions (Figure 7c).
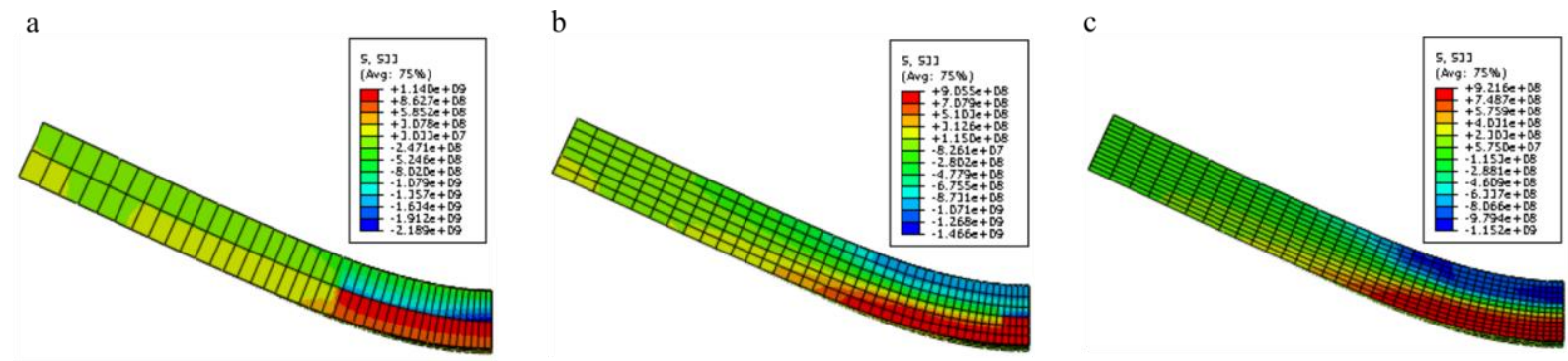

Figure 7-Through thickness stress distributions in the symmetry plane perpendicular to the bending line for the solid formulation: a) two elements through the thickness; $\boldsymbol{b}$ ) six elements through the thickness; $c$ ) twelve elements through the thickness.

Figure 8 shows the results of the difference between calculations with different numbers of elements through the thickness for the plane model. Even with 20 elements over the thickness, the results do not 
show stable behavior, and the difference $\Delta$ remains on the edge of $1 \%$. Furthermore, there is no convergence to a certain value of springback for this model (Figure 9) even with a significant number of elements through the thickness. Obviously, the plane model also does not take into account 3D effects, which need to be included for the correct prediction of the bending parameters.

As Figure 8 and Figure 9 reveal, the plane model does not provide a stable bending solution. Note that, given the "correct" mesh parameters (10 or 12 elements through the thickness) this model can deliver acceptable results. However, given its instability, this modeling approach was excluded from further discussion and the remainder of this paper focuses solely on the shell and solid models. These models, in contrast with the plane model, provide consistent results with regards to the elements/integration points through the thickness of a plate (Figure 9).

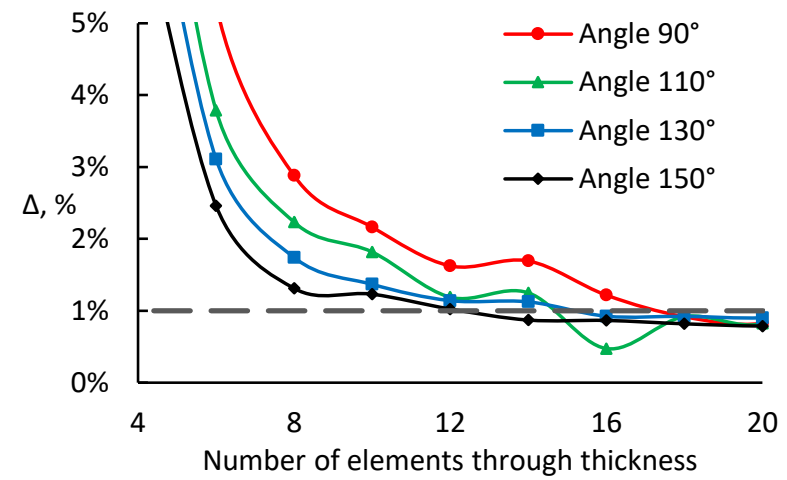

Figure 8 - Percentage difference as function of the number of elements through thickness for the plane model. Even for more than 16 elements the model does converge

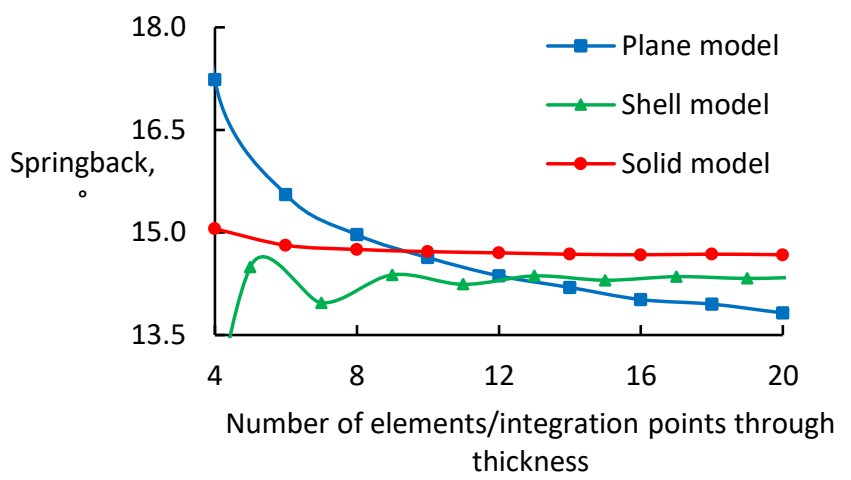

Figure 9 - Springback values for different element formulations. The plane model does not converge to a certain value even with a significant number of elements through the thickness, whereas solid and shell models do

In analogy with the elements/integration points through thickness, the same procedure has been used for the number of elements perpendicular to the bending line or along the plate length. The number of elements significantly affects the results in this case, since knowledge of the exact loading scheme during bending is of cardinal importance. The results of this analysis are presented in Figure 10 and Figure 11. Forty-five elements along the length have been selected for both models, and were used further in the mesh selection procedure. This value leads to the required percentage difference of $2.5 \%$.

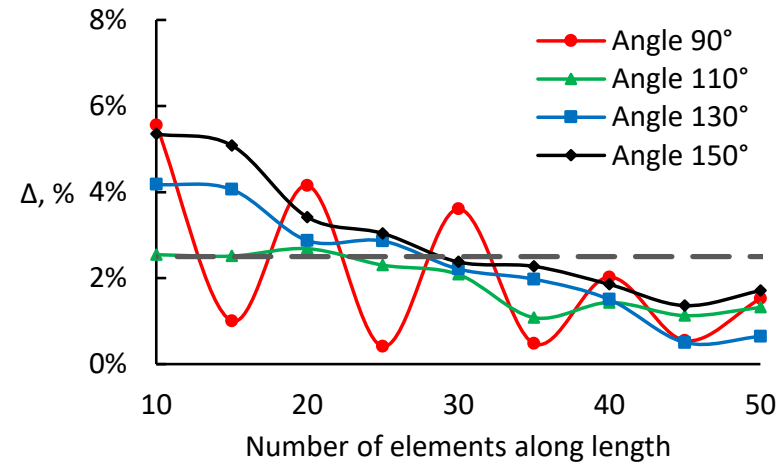

Figure 10 - Percentage springback difference for the number of elements along the length for the shell model. Mesh with 35 elements is sufficient for a difference of less than $2.5 \%$

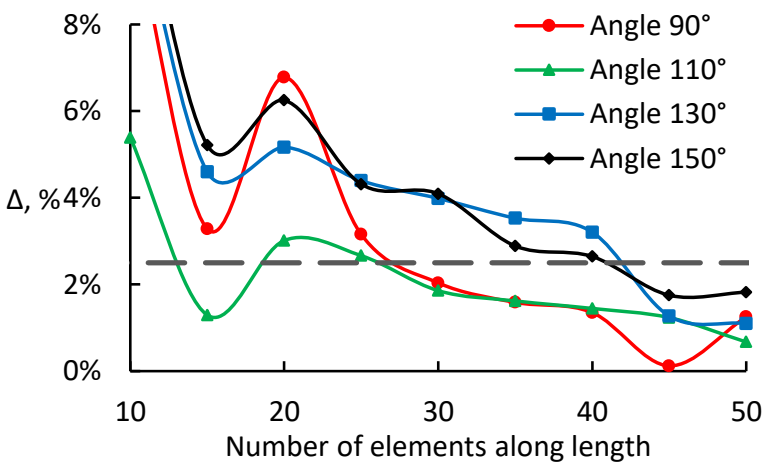

Figure 11 - Percentage springback difference for the number of elements along the length for the solid model. In this case 45 elements are necessary to achieve $2.5 \%$ 
Next the number of elements along the width were investigated. As expected, the sensitivity of the results for this mesh parameter is limited since the stress state does not change a lot along the width of a plate, despite the limited zone near the edges where the anticlastic effect takes place [17]. Figure 12 demonstrates the anticlastic effect by showing the displacement plot of the shell model. Five elements along the width are selected, and this is sufficient to assure a springback difference value below $2.5 \%$.

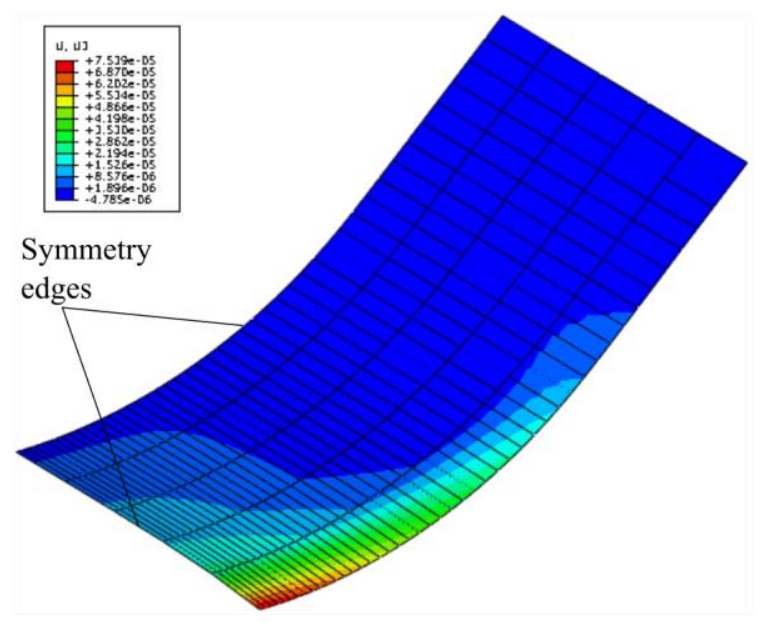

Figure 12 - Displacements of the plate in the direction of the bend line for the shell model. The anticlastic phenomenon affects only a limited region near the edge of the plate.

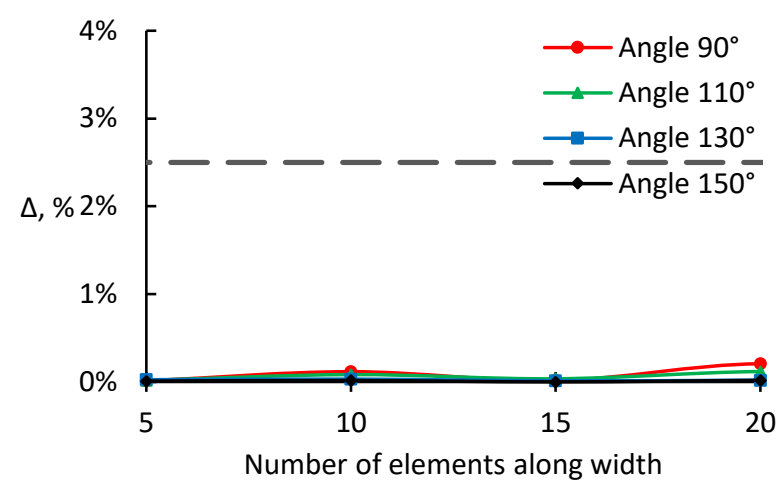

Figure 13 - Percentage springback difference as function of the number of elements along the width for the shell model. 5 elements are enough to assure a value below $2.5 \%$

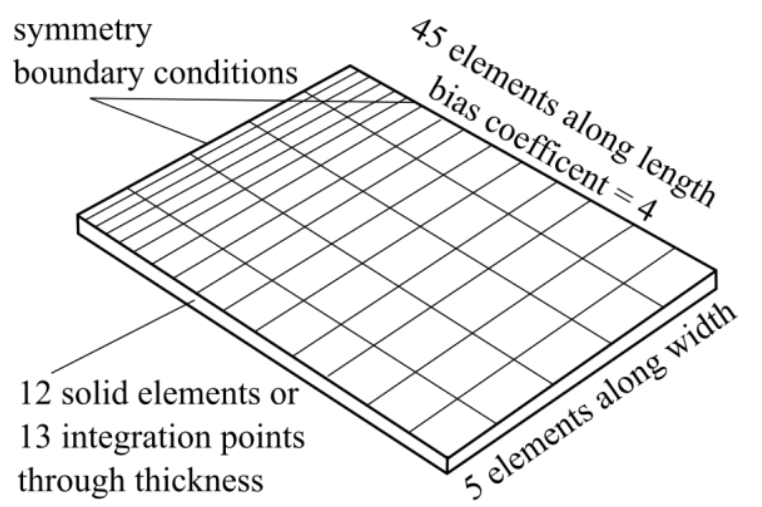

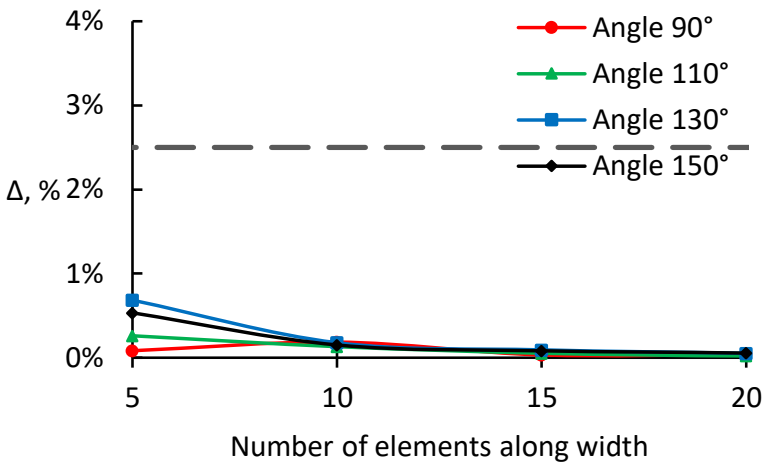

Figure 14 - Percentage springback difference as function of the number of elements along the width for the solid model. 5 elements are enough to assure a value below $2.5 \%$

Figure 15 - Final mesh parameters based on the sensitivity analysis. Bias coefficient means how many times the length of the element at one edge is greater than on the other edge. 
In this section, the mesh parameters have been selected based on the percentage springback difference (Equation 1). Figure 15 presents the final values of the mesh parameters for which adequate convergence is reached. Another important part of a finite element analysis is the correct implementation of the material behavior. We discuss the material modeling in the next section.

\section{$4 \quad$ Material modeling}

In general, the characterization of high-strength steels is a challenging task due to limited deformability and high forces required for testing. Moreover, literature provides very limited information about the considered high-strength steels: Strenx 1300 and Strenx 700 MC. To have at least some data, tensile tests have been performed. The detailed information about the tensile testing procedure is presented in [9]. The tensile tests show only limited difference in properties with respect to the rolling direction, thus, a single tensile curve is chosen for the further modeling for each material-thickness pair. For all implemented models the Poisson's ratio is 0.3. Figure 16 and Figure 17 present results of this experimental campaign for Strenx 1300 and Strenx 700 MC.

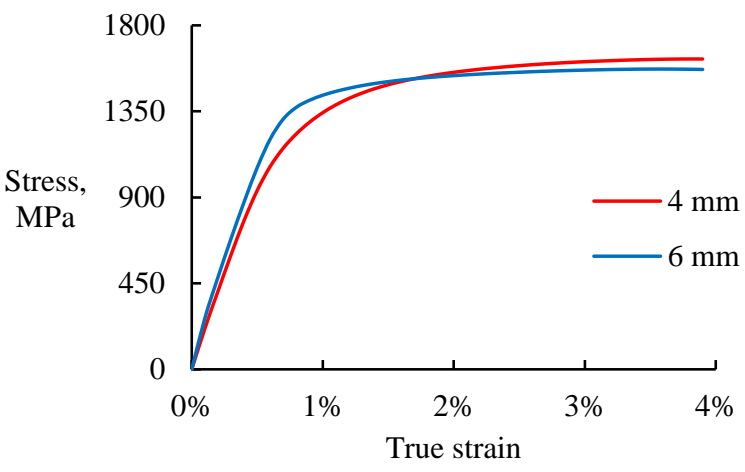

Figure 16 - Stress-strain curves for Strenx 1300

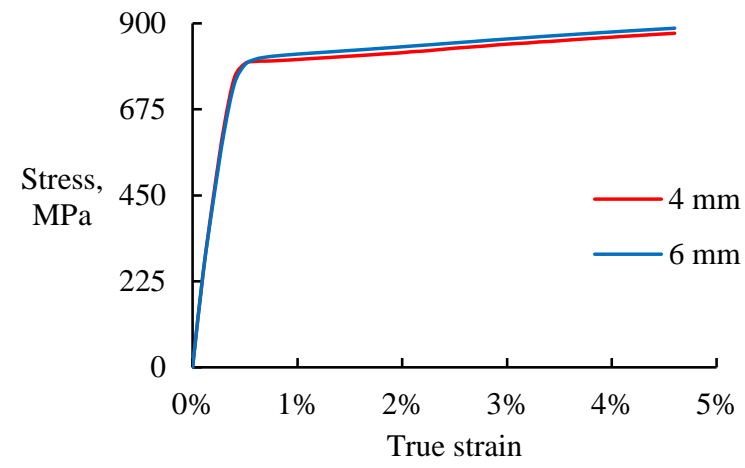

Figure 17-Stress-strain curves for Strenx 700 MC

Another important parameter for the correct prediction of bending characteristics is the change of the elasticity modulus due to prestraining $[18,19]$. In order to obtain these data, cyclic tensile testing was done: a repetitive tensile testing after various amounts of pre-straining. Figure 18 and Figure 19 provide an overview of the evolution of the elasticity modulus with an increase of logarithmic strain. Table 1 summarizes the material characteristics obtained by the tensile testing. The slightly lower value of the elasticity modulus for Strenx 1300 of $4 \mathrm{~mm}$ in comparison with $6 \mathrm{~mm}$ is due to the usual scatter of material properties for different batches of material.

Table 1

Material parameters obtained from tensile testing

\begin{tabular}{ccccc} 
Material & $\begin{array}{c}\text { Elasticity } \\
\text { modulus, GPa }\end{array}$ & $\begin{array}{c}\text { Yield stress, } \\
\text { MPa }\end{array}$ & $\begin{array}{c}\text { Maximal } \\
\text { strain, \% }\end{array}$ & $\begin{array}{c}\text { Ultimate } \\
\text { strength, MPa }\end{array}$ \\
\hline Strenx 1300 $(4 \mathrm{~mm})$ & 195 & 939.6 & 3.9 & 1623.9 \\
Strenx 1300 $(6 \mathrm{~mm})$ & 200 & 1257.2 & 3.9 & 1569.3 \\
Strenx 700 MC $(4 \mathrm{~mm})$ & 205 & 681.6 & 4.6 & 874.2 \\
Strenx 700 MC $(6 \mathrm{~mm})$ & 205 & 724.0 & 4.6 & 887.3
\end{tabular}

For any forming simulation with Abaqus it is necessary to define the plastic stress-strain curve in addition to the elastic parameters. The elastic parameters (i.e. the elasticity modulus and the yield stress) for all model executed in the current contribution have been set according to Table 1 . These 
parameters define the border after which the plastic behavior is occurred. The plastic part has been calculated according to the predefined hardening laws as presented in Section 5 and Section 7.

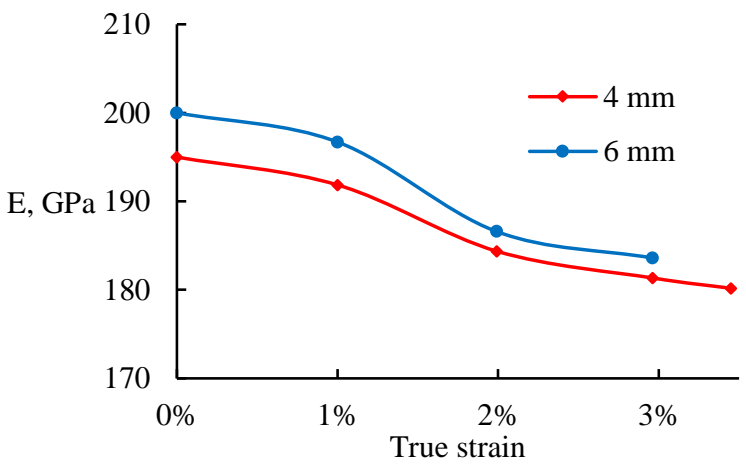

Figure 18-Change of elasticity modulus with prestrain for Strenx 1300

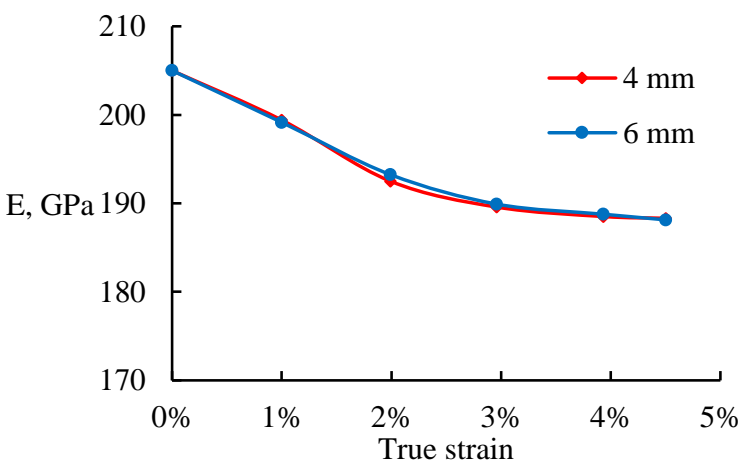

Figure 19 - Change of elasticity modulus with prestrain for Strenx $700 \mathrm{MC}$

Many researchers argue that an isotropic hardening model gives acceptable results in the prediction of forming characteristics, for instance Eggertsen and Mattiasson $[12,20]$ claim this for DP600 which is a comparable steel with Strenx $700 \mathrm{MC}$ considered in this contribution. The finite element model developed in the current contribution utilizes isotropic hardening with piecewise approximation of the stress-strain curve. Implementation of the variable elasticity modulus may lead to improved accuracy. This behavior was implemented by means of subroutines written in Fortran within the Abaqus programming environment.

\section{Results with original material parameters}

The objective of finite element model development is to cover a range of process settings, for different materials, different tool geometries and different plate thicknesses. This section provides an overview of the comparison between executed finite element models and experimental data. The complete experimental data can be found in [21] and the detailed description of the testing procedure in [1]. In total a comparison has been performed for two materials, two thicknesses, four die openings, four punch radii, and four bending angles, which results in 167 comparisons for physically feasible cases i.e. no cracking of the plate occurred and the punch radius was less than two times the die opening. Further, in graphs the results for models with the original material parameters are depicted as "Original".

For the comparison, a number of quality indicators have been selected. It is common practice to determine the coefficient of determination $R^{2}$ as the measure of the prediction quality. The coefficient of determination can be interpreted as the likelihood of the future tests falling within the predicted range. $R^{2}$ provides valuable information, however it lacks intuitive feeling. Therefore, two additional parameters are calculated: the maximal absolute error $\varepsilon_{\max }$ (Equation 2) and the average absolute error $\varepsilon_{a v}$ (Equation 3). The maximal absolute error is the absolute value of the maximal difference between the predicted and experimental values, and the average absolute error is correspondingly the average value of this difference over all comparisons. 


$$
\begin{gathered}
\varepsilon_{\text {max }}=\max \left|v_{i}^{\text {FEM }}-v_{i}^{E x p}\right| \\
\varepsilon_{a v}=\left(\sum_{i=1}^{n}\left|v_{i}^{\text {FEM }}-v_{i}^{E x p}\right|\right) / n
\end{gathered}
$$

where $v_{i}^{F E M}$ and $v_{i}^{E x p}$ are finite element and experimental data correspondingly, $n$ represents the number of comparisons.

Figure 20 and Figure 21 show examples of the results comparison between the experimental data and the finite element models.

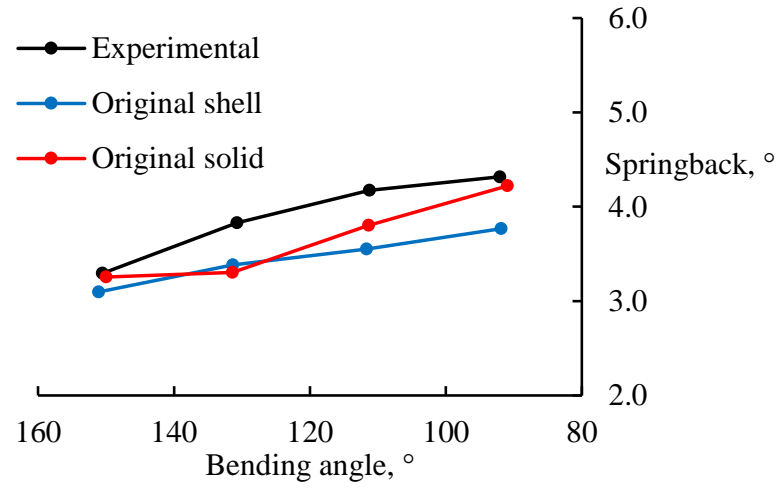

Figure 20-Comparison of results for finite element model with experimental data. Models: shell and solid; plate: Strenx $700 \mathrm{MC}$; thickness: $6 \mathrm{~mm}$; die opening: $40 \mathrm{~mm}$; punch radius: $10 \mathrm{~mm}$

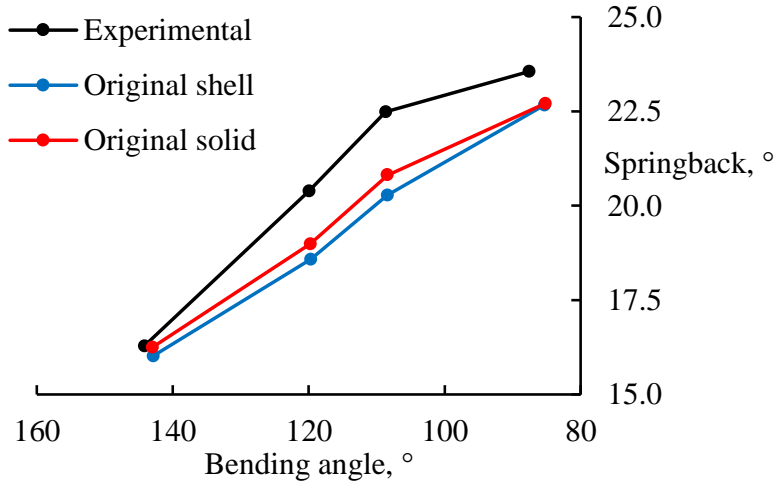

Figure 21 - Comparison of results for finite element model with experimental data. Model: shell and solid; plate: Strenx 1300; thickness: $4 \mathrm{~mm}$; die opening: $80 \mathrm{~mm}$; punch radius: $30 \mathrm{~mm}$

Table 2 provides an overview of the comparative parameters. In general, the finite element model gives acceptable results for the contact points position, springback angle and bend allowance. However, for the bending force, the prediction is inacceptable: $R^{2}$ values for the bending force are quite low, thus the finite element model in the status does not predict this bending characteristic well.

Table 2

\begin{tabular}{|c|c|c|c|c|c|}
\hline Model type & $\begin{array}{c}\text { Comparison } \\
\text { parameter }\end{array}$ & $\begin{array}{l}\text { Contact points } \\
\text { position, } \mathrm{mm}\end{array}$ & $\begin{array}{c}\text { Springback } \\
\text { angle, }^{\circ}\end{array}$ & $\begin{array}{c}\text { Bending } \\
\text { force, } \mathrm{kN} / \mathrm{m}\end{array}$ & $\begin{array}{c}\text { Bend } \\
\text { allowance, } \mathrm{mm}\end{array}$ \\
\hline \multirow{4}{*}{ Shell } & $R^{2}$ & 0.9710 & 0.9732 & 0.7936 & 0.8793 \\
\hline & $\varepsilon_{a v}$ & 0.9051 & 0.9774 & 316.94 & 0.8416 \\
\hline & $\varepsilon_{\max }$ & 4.9133 & 5.0951 & 2144.1 & 7.4935 \\
\hline & $R^{2}$ & 0.9682 & 0.9713 & 0.8298 & 0.8688 \\
\hline \multirow[t]{2}{*}{ Solid } & $\varepsilon_{a v}$ & 1.0332 & 0.8388 & 313.75 & 0.8849 \\
\hline & $\varepsilon_{\max }$ & 5.5339 & 5.7879 & 2153.5 & 7.5128 \\
\hline
\end{tabular}

Overview of comparison of original finite element model with experimental data

The maximal error for shell and solid models of springback is more than $5^{\circ}$, which is quite inaccurate. These maximal errors are for the case of a die opening of $80 \mathrm{~mm}$ and a punch radius of $40 \mathrm{~mm}$. In the last stage of the forming in this configuration, the die already penetrates the bent plate (Figure 22). The finite element model is not able to take this phenomenon into account. Figure 23 shows that for the smaller angles the finite element model delivers acceptable results, but fails to predict the last stage correctly where indentation already takes place. 

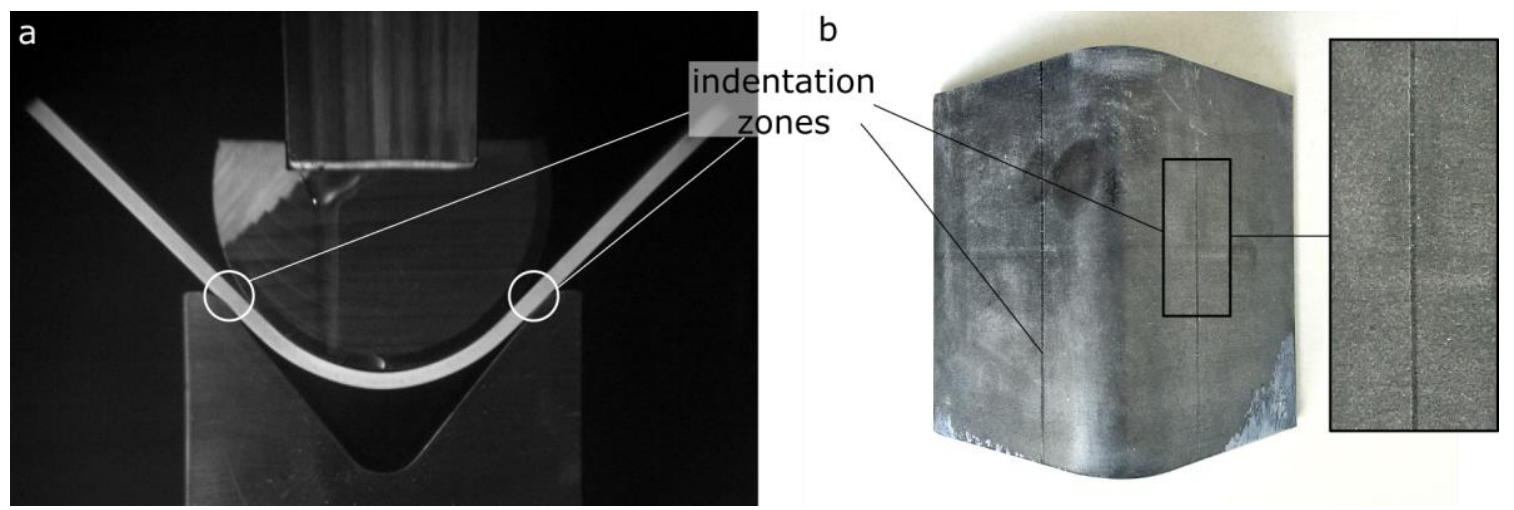

Figure $22-\boldsymbol{a})$ The die penetrates the plate during the last stage of the forming process; $\boldsymbol{b})$ view on the bottom of the plate after the forming process reveals the indentation zones. Die opening: $80 \mathrm{~mm}$; punch radius $40 \mathrm{~mm}$; plate thickness: 4 mm; material: Strenx $700 \mathrm{MC}$.

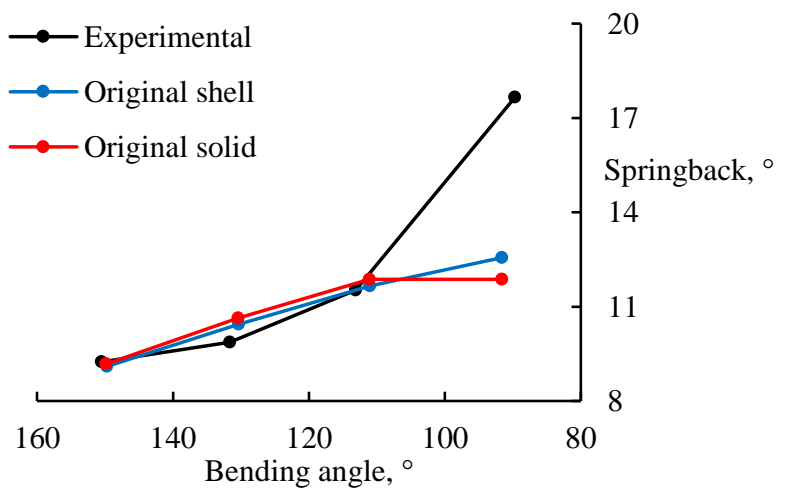

Figure 23 - Comparison of results for the finite element model with experimental data. Model: shell and solid; plate: Strenx 700 MC; thickness: $4 \mathrm{~mm}$; die opening: $80 \mathrm{~mm}$; punch radius: $40 \mathrm{~mm}$

\section{Aerens hardening law}

The Swift model is the most commonly used material law for description of the hardening behavior of steels. It approximates the hardening behavior as the stress response $\sigma$ in the function of strain $\varepsilon$ in a form of:

$$
\sigma=K_{S} \cdot\left(\varepsilon+\varepsilon_{0 S}\right)^{n_{S}}
$$

where $K_{s}$ is the strength coefficient, $\varepsilon_{0 \text { s }}$ the initial deformation, $n_{s}$ the hardening exponent.

However, in the case of high-strength steels the Swift law fails to accurately approximate the stressstrain curve, as shown in Figure 24 and Figure 25. Therefore, a better constitutive model is needed for high-strength steels. The Aerens law, which is a modified Swift law, provides an approximation of the stress-strain curve in a form of:

$$
\sigma=\left[1-e^{k \cdot\left(\varepsilon-\varepsilon_{e l}\right)}\right]\left[K_{A} \cdot\left(\varepsilon+\varepsilon_{0 A}\right)^{n_{A}}-\sigma_{e l}\right]+\sigma_{e l}
$$

where $K_{A}, \varepsilon_{O A}, n_{a}$ have the same meaning as for the Swift law, and $k$ is defined as:

$$
k=\frac{E}{K_{A} \cdot\left(\varepsilon_{e l}+\varepsilon_{0 A}\right)^{n} A-\sigma_{e l}}
$$

Figure 24 and Figure 25 show the best fit results for the Swift and Aerens models for two selected highstrength steels. The fitting has been done for the interval between $0 \%$ and maximal logarithmic strain 
depending on the material (Table 1). Table 3 summarizes the fitting error by presenting the root mean square error RMSE between predicted and experimentally observed values.

Table 3

Root mean square error RMSE for the fitting of tensile data with Swift and Aerens laws

\begin{tabular}{cll} 
Material & Model & \multicolumn{1}{c}{ RMSE } \\
\hline Strenx 1300 (4 mm) & Swift & 40.52 \\
& Aerens & 1.46 \\
Strenx 1300 (6 mm) & Swift & 7.14 \\
& Aerens & 6.31 \\
Strenx 700 MC (4 mm) & Swift & 50.24 \\
& Aerens & 35.43 \\
Strenx 700 MC (6 mm) & Swift & 62.87 \\
& Aerens & 19.34
\end{tabular}

Based on the values of RMSE from Table 3, the Aerens law provides a rather accurate match with the measured data, whereas the Swift law in the form of Equation 4 does not take into account the smooth transition between the elastic and plastic zones of high strength steels and thus provides a less accurate approximation. Equation 5 looks more refined than Equation 4, however it requires the same parameters as the Swift model and in addition, the strain and the stress at the elastic limit $\varepsilon_{e l}$ and $\sigma_{e l}$ respectively. Table 4 presents the best fit parameters for the Swift and Aerens hardening laws. The difference in the material parameters for the same material with different thicknesses in Table 4 is due to the natural scatter of the material properties, since the testing material has been taken from different batches.

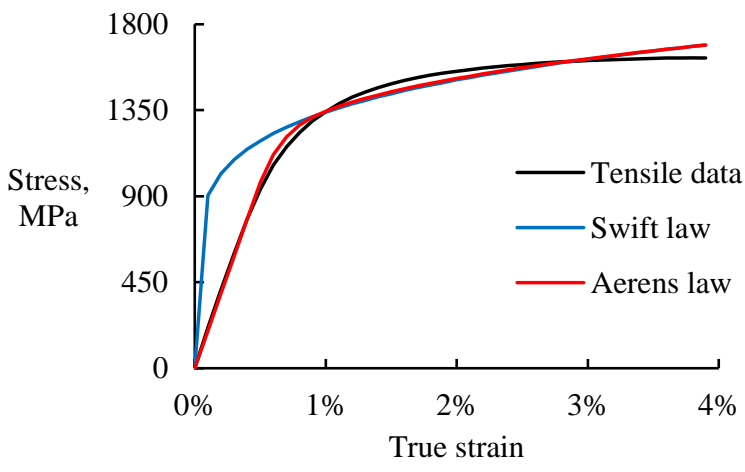

Figure 24-The Swift law fails to approximate the smooth transition zone between the elastic and plastic zones for Strenx $13004 \mathrm{~mm}$, whereas the Aerens law delivers an accurate approximation of complete stress-strain curve

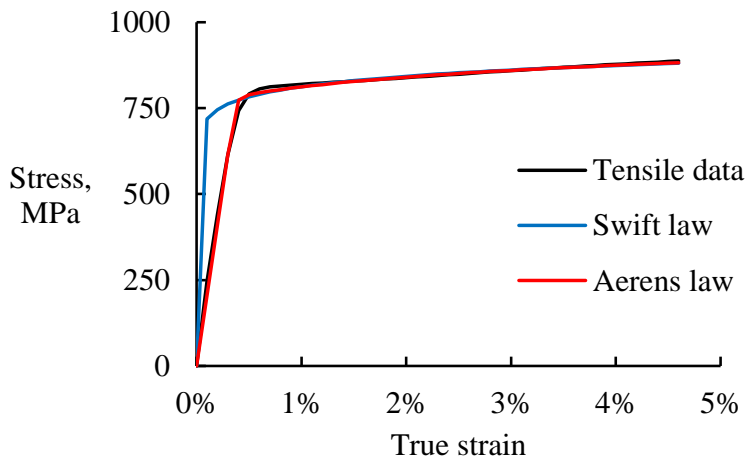

Figure 25 - The Swift law fails to approximate the smooth transition zone between the elastic and plastic zones for Strenx $700 \mathrm{MC} 4 \mathrm{~mm}$, whereas the Aerens law delivers an accurate approximation of complete stress-strain curve

Table 4

Overview of material parameters for Swift and Aerens hardening laws

\begin{tabular}{ccccccc} 
Material & $K_{S}, \mathrm{MPa}$ & $\varepsilon_{O S}$ & $n_{S}$ & $K_{A}, M P a$ & $\varepsilon_{O A}$ & $n_{A}$ \\
\hline Strenx 1300 $(4 \mathrm{~mm})$ & 2946 & 0.000 & 0.171 & 2860 & 0.000 & 0.163 \\
Strenx 1300 (6 mm) & 2076 & 0.000 & 0.080 & 2043 & 0.000 & 0.076 \\
Strenx 700 MC (4 mm) & 1327 & 0.000 & 0.125 & 1741 & 0.159 & 0.434 \\
Strenx 700 MC (6 mm) & 1037 & 0.000 & 0.053 & 1080 & 0.005 & 0.068
\end{tabular}




\section{Adapted material parameters}

In this section the described model is further improved by reverse engineering the constitutive law based on the experimental results. The Aerens law (Equation 5) is used for this purpose. As it was already discussed in Section 3, the springback is the most important bending characteristic. Therefore, in this section, the minimization of the difference between experimental and predicted values for springback is implemented, and this adjustment is expected to improve prediction accuracy. The error minimization is implemented only for a limited training set ( 3 test cases) of the tooling combinations, then the calculation is provided for the complete validation set (11 test cases) in order to verify the usability and robustness of the obtained material parameters.

Analysis of results obtained by the simulations shows that the springback values always underestimate the experimental data (Figure 20 and Figure 21). This can be caused by different sources, such as over simplistic representation of the contact interaction, hardening mechanism, or use of the tensile testing curve instead of unit moment curve [2]. This error can be compensated by identifying updated values for the material parameters in the Aerens law, namely the two most important constants: $K_{A}$ and $n_{A}$.

For the search of adapted material parameters, the following procedure was used. Three test cases were selected with the following tooling combinations: die opening $80 \mathrm{~mm}$ with punch radius $40 \mathrm{~mm}$; die opening 60 with punch radius $20 \mathrm{~mm}$; and die opening $40 \mathrm{~mm}$ with punch radius $10 \mathrm{~mm}$. These cases represent three different ratios of punch radius/die opening, which defines the type of bending [1]. It was assumed that, since this selection covers a wide range of process parameter combinations, the derived corrections for the material parameters will lead to a significant improvement for the other test cases as well. Simulations are run for the above mentioned cases for different $K_{A}$ and $n_{A}$. Afterwards the error for one test case is calculated according to:

$$
\epsilon=\sqrt{\sum_{i=1}^{n=4}\left(\Delta \beta_{i}^{\text {exp }}-\Delta \beta_{i}^{\text {sim }}\right)^{2}}
$$

where $\Delta \beta_{i}^{\text {exp }}$ and $\Delta \beta_{i}^{\text {sim }}$ are springback values for angles $90^{\circ}, 110^{\circ}, 130^{\circ}$ and $150^{\circ}$.

The total error value is calculated by summation of the errors for the three considered test cases as:

$$
\epsilon_{\text {tot }}=\epsilon_{80-40}+\epsilon_{60-20}+\epsilon_{40-10}
$$

where $\epsilon_{80-40}$ is the error for the case of die opening $80 \mathrm{~mm}$ and punch radius $40 \mathrm{~mm} ; \epsilon_{60-20}$ is the error for the case of die opening $60 \mathrm{~mm}$ and punch radius $20 \mathrm{~mm} ; \epsilon_{40-10}$ is the error for the case of die opening $40 \mathrm{~mm}$ and punch radius $10 \mathrm{~mm}$.

Test simulations are executed for a number of $K_{A}$ and $n_{A}$ combinations with a predefined steps (100 for $K_{A}, 0.005$ for $n_{A}$ ) starting from the initial material parameters. Further, based on the minimal error criteria, the new parameters are selected from the obtained test simulations for all considered combinations materials/thicknesses, which are further used in the finite element calculations. Figure 26 and Figure 27 represent examples of the error graphs with respect to $n_{A}$ and $K_{A}$ for Strenx $13004 \mathrm{~mm}$ and $6 \mathrm{~mm}$. The values of updated parameters are presented in Table 5. 


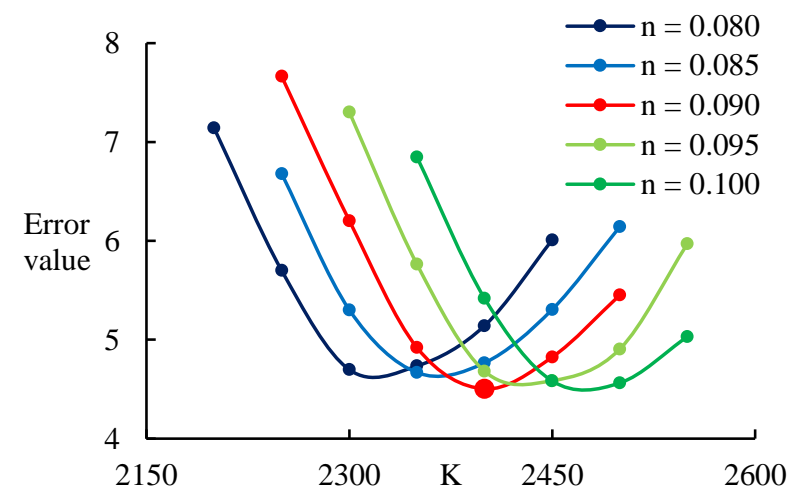

Figure 26 - Error values for the case of Strenx $13004 \mathrm{~mm}$

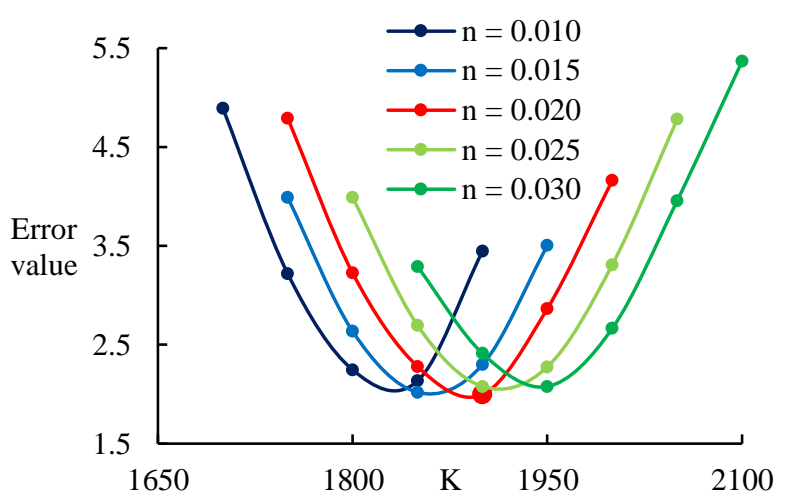

Figure 27-Error values for the case of Strenx $13006 \mathrm{~mm}$

Table 5

Adapted parameters for the Aerens law

\begin{tabular}{cccl} 
Material & $\bar{K}_{A}, M P a$ & $\bar{\varepsilon}_{0 A}$ & $\bar{n}_{A}$ \\
\hline Strenx 1300 $(4 \mathrm{~mm})$ & 2400 & 0.0000 & 0.09 \\
Strenx 1300 $(6 \mathrm{~mm})$ & 1900 & 0.0000 & 0.02 \\
Strenx 700 MC $(4 \mathrm{~mm})$ & 3100 & 0.0033 & 1.10 \\
Strenx 700 MC $(6 \mathrm{~mm})$ & 3600 & 0.0035 & 1.65
\end{tabular}

It is expected that adapted material parameters improve the prediction quality. In the next section, the results with adapted material parameters are presented and compared with the experimental data.

\section{Results with adapted material parameters}

This section gives an overview of results with the adapted material parameters according to the Aerens law. As in Section 5, Table 6 provides a comparison with experimental data by listing $R^{2}, \varepsilon_{\text {max }}$ and $\varepsilon_{a v}$. In general, the quality of prediction is better with the introduction of the adapted material parameters. $R^{2}$ increased, $\varepsilon_{\max }$ and $\varepsilon_{a v}$ decreased for all bending characteristics except the contact points position. This deviation is due to the fact that the experimental measurement procedure determines the border of the contact zone (for more details the reader can refer to [1]), whereas in the finite element model an exact line with the maximum contact pressure is taken into consideration. Further, in graphs the results for models with the adapted material parameters are depicted as "Adapted".

Table 6

Overview of comparison of adapted finite element model with experimental data. $\uparrow$ - represents increased prediction quality for a bending characteristic with respect to the initial finite element model, and $\downarrow$ - decreased prediction quality.

\begin{tabular}{cccccc} 
Model type & $\begin{array}{c}\text { Comparison } \\
\text { parameter }\end{array}$ & $\begin{array}{c}\text { Contact points } \\
\text { position } \downarrow\end{array}$ & $\begin{array}{c}\text { Springback } \\
\text { angle } \uparrow\end{array}$ & $\begin{array}{c}\text { Bending } \\
\text { force } \uparrow\end{array}$ & $\begin{array}{c}\text { Bend } \\
\text { allowance } \uparrow\end{array}$ \\
\hline \multirow{3}{*}{ Shell } & $R^{2}$ & 0.9663 & 0.9778 & 0.8053 & 0.9000 \\
& $\varepsilon_{a v}$ & 1.7462 & 0.5402 & 279.96 & 0.7294 \\
& $\varepsilon_{\max }$ & 5.7226 & 4.7744 & 1951.6 & 7.0355 \\
Solid & $R^{2}$ & 0.9683 & 0.9749 & 0.8434 & 0.9011 \\
& $\varepsilon_{a v}$ & 1.5609 & 0.6467 & 257.26 & 0.7308 \\
& $\varepsilon_{\max }$ & 5.7361 & 5.1475 & 941.204 & 6.4597
\end{tabular}

Figure 28 provides an example of the experimental data compared with original and adapted material parameters for the shell formulation, and Figure 29 does so for the solid formulation. The predicted values for the adapted model are closer to the experimental data. 


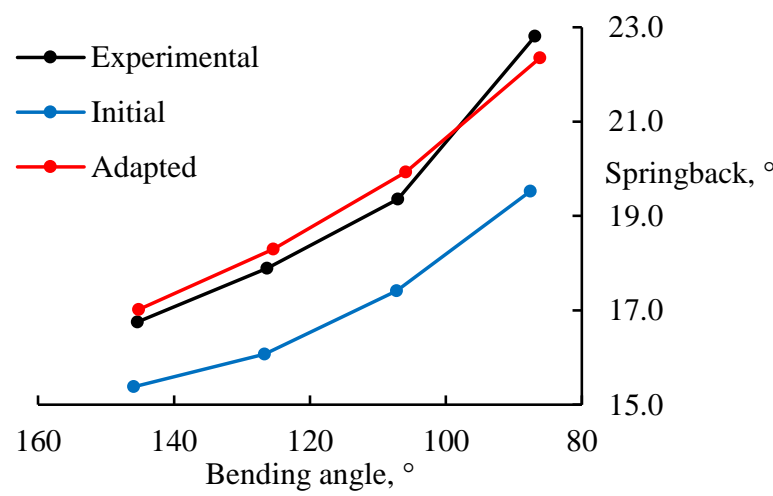

Figure 28-Comparison of results for original and adapted finite element models with experimental data. Model: shell; plate: Strenx 1300; thickness: $4 \mathrm{~mm}$; die opening: $80 \mathrm{~mm}$; punch radius: $10 \mathrm{~mm}$

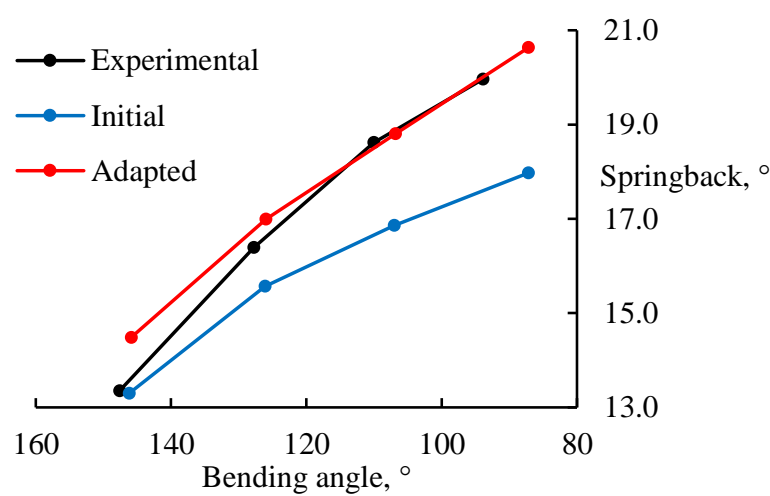

Figure 29-Comparison of results for original and adapted finite element models with experimental data. Model: solid; plate: Strenx 1300; thickness: $4 \mathrm{~mm}$; die opening: $60 \mathrm{~mm}$; punch radius: $30 \mathrm{~mm}$

\section{Conclusions}

Finite element modeling is a powerful approach for the prediction of large radius bending. Unlike the plane model, solid and shell formulations provide a stable prediction of the forming process if model parameters are set properly. Additionally, an optimal selection of mesh parameters is required in order to combine stability and accuracy of the results with a minimized solution time.

An accurate description of the material model has also a significant influence on the prediction quality. The typical approach is to use the stress-strain curve obtained by tensile testing as the description of the hardening behavior. However, better results can be achieved using an appropriate hardening law. The Aerens model provides a suitable approximation of the stress-strain curve for high-strength steels and offers an opportunity to identify optimal material parameters for the finite element model. These optimal parameters lead to increased accuracy of the prediction.

Solid and shell formulations both deliver an acceptable prediction quality. Nevertheless, the solid model gives a more complete description of the considered problem, by modeling not only the mid-surface as in the case of the shell formulation. This more complete model has an apparent drawback in the increased calculation time with comparison to shell formulation.

All simulation results can be found in the Dataverse research data repository [22], along with the complete experimental data [21]. These data can be further used as benchmark for future improvements on the prediction of large radius bending of high-strength steels by diverse prediction methods.

\section{References}

1. Vorkov V, Aerens R, Vandepitte D, Duflou JR (2017) Experimental investigation of large radius air bending. Int J Adv Manuf Technol 92 (9):3553-3569

2. Aerens R, Masselis S (2000) Air bending. Scientific and Technical Research Center of the Metal Fabrication Industry (CRIF/WTCM/SIRRIS) MC 110, Leuven, Belgium

3. De Vin LJ, Streppel AH, Singh UP, Kals HJJ (1996) A process model for air bending. J Mater Process Technol 57 (1-2):48-54 
4. Yang X, Choi C, Sever NK, Altan T (2016) Prediction of springback in air-bending of advanced high strength steel (DP780) considering Young's modulus variation and with a piecewise hardening function. Int J Mech Sci 105:266-272

5. Teimouri R, Baseri H, Rahmani B, Bakhshi-Jooybari M (2014) Modeling and optimization of spring-back in bending process using multiple regression analysis and neural computation. Int J Mater Form 7

(2):167-178

6. Vorkov V, Aerens R, Vandepitte D, Duflou JR (2017) Accurate prediction of large radius air bending using regression. Procedia Eng 207:1623-1628

7. Fu Z, Mo J, Chen L, Chen W (2010) Using genetic algorithm-back propagation neural network prediction and finite-element model simulation to optimize the process of multiple-step incremental airbending forming of sheet metal. Mater Des 31 (1):267-277

8. Vorkov V, Aerens R, Vandepitte D, Duflou JR (2015) On the identification of a loading scheme in large radius air bending. Key Eng Mater 639:155-162

9. Vorkov V, Aerens R, Vandepitte D, Duflou JR (2014) Springback prediction of high-strength steels in large radius air bending using finite element modeling approach. Procedia Eng 81:1005-1010

10. Ledentsov D, Düster A, Volk W, Wagner M, Heinle I, Rank E (2010) Model adaptivity for industrial application of sheet metal forming simulation. Finite Elem Anal Des 46 (7):585-600

11. Arola A-M, Mäntyjärvi K, Karjalainen JA (2013) FEM-modeling of bendability of ultra-high strength steel. Key Eng Mater 549:333-339

12. Eggertsen P-A, Mattiasson K (2011) On the identification of kinematic hardening material parameters for accurate springback predictions. Int J Mater Form 4 (2):103-120

13. Panthi SK, Ramakrishnan N, Ahmed M, Singh SS, Goel MD (2010) Finite element analysis of sheet metal bending process to predict the springback. Mater Des 31 (2):657-662

14. SSAB (2017) Strenx 1300 Data Sheet. Retrieved from https://www.ssab.com/products/brands/strenx/products/strenx-1300, on 24.12.2017

15. SSAB (2017) Strenx 700 MC Data Sheet. Retrieved from https://www.ssab.com/products/brands/strenx/products/strenx-700-mc, on 24.12.2017

16. Wagoner RH, Li. M (2007) Simulation of springback: through-thickness integration. Int J Plast 23:345360

17. Horrocks D, Johnson W (1967) On anticlastic curvature with special reference to plastic bending: a literature survey and some experimental investigations. Int J Mech Sci 9 (12):835-861

18. Chatti S, Fathallah R (2012) A study of the variations in elastic modulus and its effect on springback prediction. Int J Mater Form:1-11

19. Wagoner RH, Lim H, Lee M-G (2013) Advanced issues in springback. Int J Plast 45:3-20

20. Eggertsen P-A, Mattiasson K (2009) On the modelling of the bending-unbending behaviour for accurate springback predictions. Int J Mech Sci 51:547-563

21. Vorkov V (2017) Complete experimental data for large radius bending. doi:10.7910/DVN/P7XKVV, The Dataverse project

22. Vorkov V (2017) FEM data for large radius bending. doi:10.7910/DVN/WJGDUR, The Dataverse project 